\title{
Recurrent ectopic pregnancy at the ipsilateral tubal stump following total salpingectomy case report and review of literature
}

\author{
Lakhotia S, Yussof SM*, and Aggarwal I \\ Department of Obstetrics \& Gynecology, KK Women's and Children's Hospital, Singapore
}

\begin{abstract}
Ectopic pregnancy continues to be an important cause of maternal morbidity and mortality. We present a case of a 38-year-old woman who had an ectopic pregnancy occurring in the stump of a fallopian tube following ipsilateral salpingectomy for a ruptured ectopic pregnancy. This is a rare presentation for ectopic pregnancy and yet has significant consequences for the patient owing to diagnostic dilemma and increased mortality rate. It also emphasizes that salpingectomy does not exclude recurrence of ipsilateral ectopic and highlights the need for high index of suspicion. This case-report also summarises the importance of precise surgical techniques.
\end{abstract}

\section{Introduction}

Ectopic pregnancy occurs in around 1-2\% of all pregnancies [1]. The incidence of recurrent ectopic pregnancy is approximately $15 \%$ after one ectopic pregnancy and rises to $30 \%$ after two ectopic pregnancies [2]. Despite major advances in its early diagnosis and treatment, ectopic pregnancy continues to be an important cause for maternal mortality, morbidity and early pregnancy loss. A World Health Organization analysis of maternal deaths showed ectopic pregnancy to be responsible for $4.9 \%$ of all and $6.1 \%$ of direct maternal deaths in developed countries [3].

Ipsilateral ectopic pregnancy after partial or total salpingectomy is a rare occurrence with very few cases reported in the English literature in the last two decades [4-12,21-24]. This type of ectopic pregnancy is associated with increased mortality rates due to delay in diagnosis and treatment [13]. We present a rare case of ipsilateral ectopic pregnancy occurring in the stump of a previous salpingectomy site in a 38-yearold woman, who presented to the A\&E department of our hospital with lower abdominal pain.

\section{Case presentation}

A 38-year-old woman presented to the A\&E department of a tertiary hospital, with 4 weeks' amenorrhoea and a history of lower abdominal pain for one day. She had no complaints of bleeding per vaginum, dizziness or gastrointestinal symptoms. She was gravida 6 para 2, with two previous normal vaginal deliveries, two first trimester termination of pregnancies and a right sided tubal ectopic pregnancy 8 months prior, for which she had a laparoscopic total salpingectomy.

She had no significant past medical history. She was a non-smoker and was not on any contraception presently. Her past surgical history was significant for laparoscopic total salpingectomy eight months ago. Her past menstrual cycles were regular. The detailed menstrual history revealed that she had normal menstrual period 8 weeks ago lasting for 4-5 days. Around four weeks prior to this presentation in A\&E she had spotting for two days. She considered this as her last menstrual period.
On clinical examination vital signs were stable and there was no pallor. Her abdomen was soft but distended, with generalized tenderness on palpation. There was no rebound tenderness or guarding. On vaginal examination cervix was closed and there was no vaginal bleeding or discharge. Uterus was normal sized, anteverted. No adnexal masses were palpable and cervical motion tenderness was absent.

Urine hCG done in A\&E was positive. Her haemoglobin was 12.4 g/dL. Her serum Beta hCG level was 825.1IU/L. Total WBC count and urinalysis was normal. A transvaginal ultrasound scan showed empty endometrial cavity (Figure 1) and moderate hemoperitoneum in the

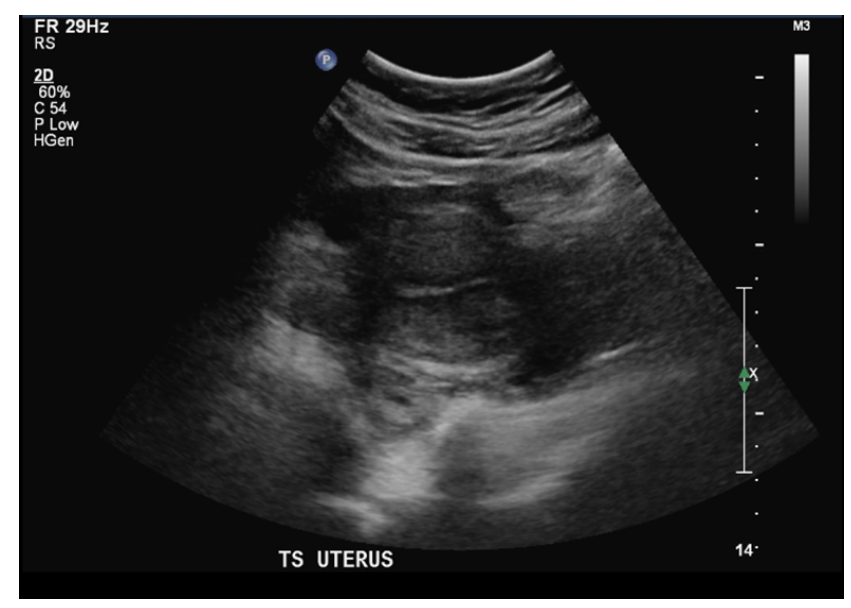

Figure 1. Empty uterus on TV ultrasonography.

Correspondence to: Shilla Mariah Yussof, Department of Obstetrics andGynaecology, KK Women's and Children's Hospital, Singapore, Tel: +65 6293 4044; E-mail: shillamariah@gmail.com

Received: August 26, 2016; Accepted: September 22, 2016; Published: September 26, 2016 
pouch of Douglas (Figure 2). There was no obvious adnexal mass seen. No corpus luteum was noted.

In view of severe pain in abdomen, hCG levels 825.1IU/L and ultrasound suggestive of moderate hemoperitoneum, the diagnosis of ruptured ectopic pregnancy in contralateral tube was suspected.

The differential diagnosis was ruptured corpus luteum cyst leading to hemoperitoneum. However, there was no corpus luteum evident on ultrasound scan.

The above clinical findings and investigations were discussed with the patient. The decision was made for a diagnostic laparoscopy followed by salpingectomy or cystectomy depending upon the intraoperative findings. Intra-operatively, she was found to have moderate hemoperitoneum and ruptured ectopic pregnancy measuring about $1 \mathrm{~cm}$ and arising from the right tubal stump at the site of previous salpingectomy. A gestational sac could clearly be seen on the surface of the stump (Figure 3). Both ovaries were normal. No corpus luteum was seen. The left tube was normal.

The blood clots were removed using suction and irrigation. To facilitate the excision of stump ectopic and reduce the intra-operative blood loss, diluted vasopressin was injected into the right cornua. The right tubal stump was then completely excised using diathermy (Figure 4) and hemostasis was achieved.

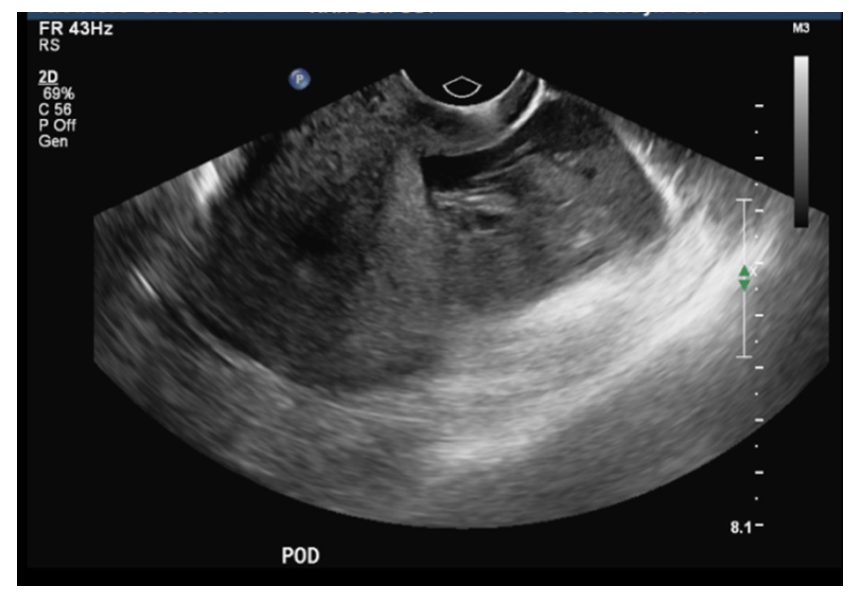

Figure 2. Hemoperitoneum in pouch of Douglas.

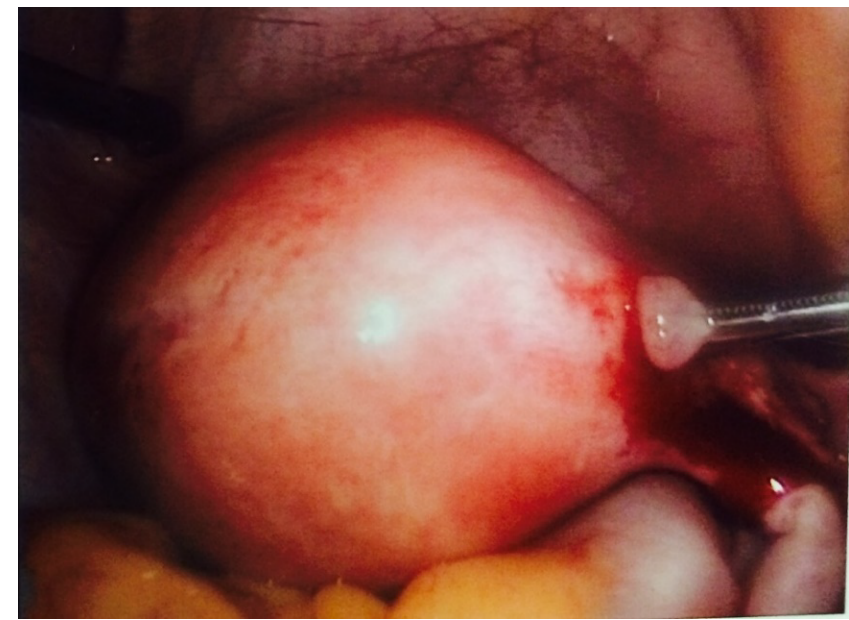

Figure 3. Gestational sac at right tubal stump.
A check hysteroscopy was performed after the excision of stump ectopic to assess the uterine cavity and rule out cornual/angular ectopic pregnancy. The uterine cavity was normal, and both ostia were visualized (Figure 5).

She was well postoperatively and was discharged the following day. The serum beta hCG level was repeated on follow up visit after two weeks and was found to be negative. Histology of the right tubal stump showed products of conception with trophoblastic cells present.

\section{Discussion}

Ectopic pregnancy has become a major health problem in reproductive age group women owing to increased prevalence of pelvic surgery, pelvic inflammatory disease and assisted reproductive techniques. It can have wide spectrum of clinical presentation and the classic triad of amenorrhea, abdominal pain and vaginal bleeding is present in only upto $50 \%$ of cases. It has been described as a "great masquerader". The variation in clinical signs and symptoms suggests that different population of people with EP may present differently, especially with recurrent EP. It remains an important cause of morbidity and mortality in women of childbearing age.

The incidence of Ectopic pregnancy is around 1-2\%. Approximately $92 \%$ of ectopic pregnancies occur in the ampullary region of the

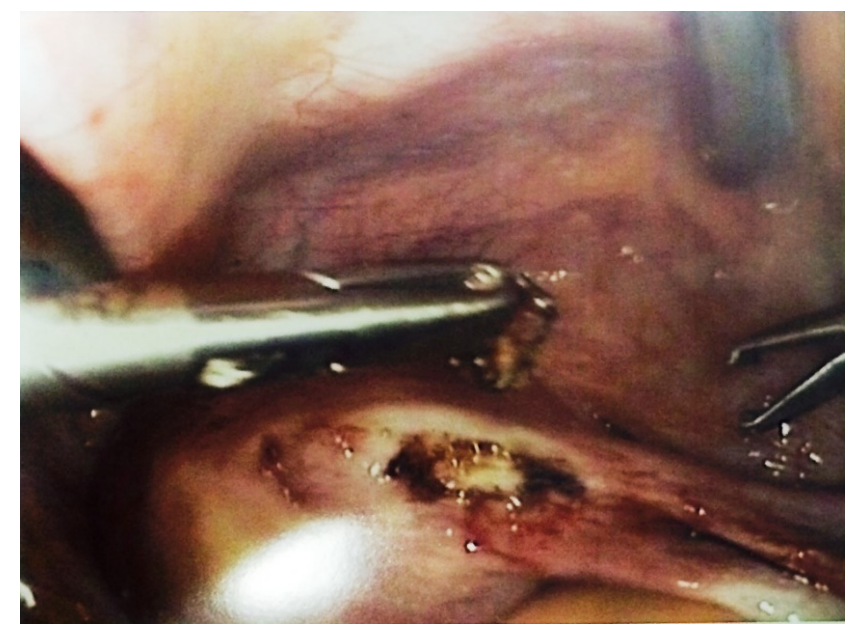

Figure 4. Excision of tubal stump.
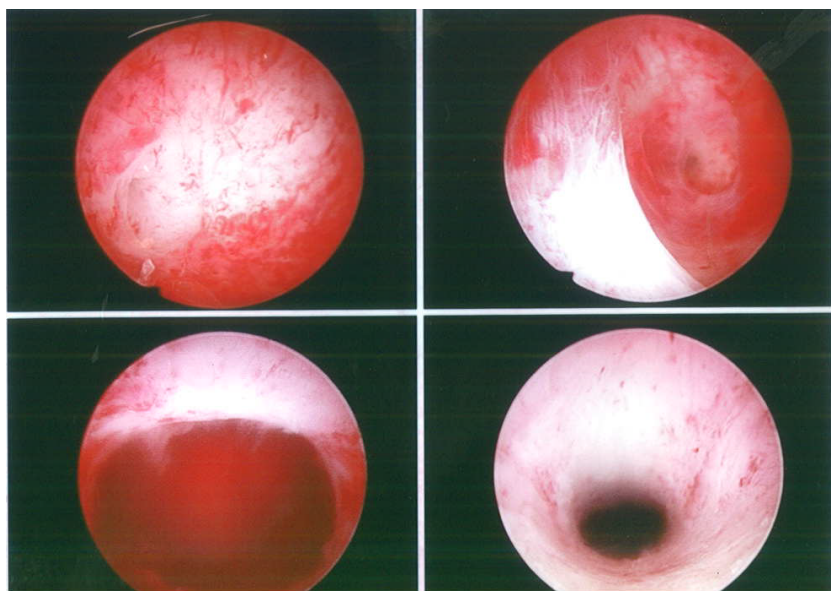

Figure 5. Hysteroscopic view. 
fallopian tubes, $2.5 \%$ as interstitial/cornual ectopic pregnancies, while less-common forms include cervical, ovarian, and peritoneal [14]. Ectopic pregnancy occurring in the remnant stump of the fallopian tube following salpingectomy is even less common. There have been isolated case reports in literature showing few cases of ectopic pregnancy in proximal or distal stump after total or partial salpingectomy or after adnexectomy. However, the exact incidence of ectopic pregnancy in the remnant stump following salpingectomy is not known currently. Takeda et al. reported an incidence of $1.16 \%$ in their department from January 1994 to August 2005 [14].

Ectopic pregnancy occurring after total salpingectomy or adnexectomy can occur in either the isthmic part of the tube (stump ectopic) or interstitial part of the tube.

Isthmic ectopic pregnancy is a gynaecological emergency, with mortality rates around $2.0-2.5 \%$ in contrast to other ectopic pregnancies where mortality rates are around $0.14 \%$ [13]. This location is associated with high risk of rupture and severe bleeding at an early gestational age. This is due to the poor distensibility of this portion of the tube and rich vascularity of the area [14].

We did a comprehensive literature search to find out sonographic features which would clearly distinguish between an interstitial pregnancy, an angular pregnancy, pregnancy in isthmic remnant of the tube or cornual pregnancy. However, the ultrasound signs are subtle and confusing with no clear differentiating features amongst these. The use of high-resolution transvaginal sonography or 3D sonography can be of aid in obtaining the coronal scans of fundal region of the uterus thus giving more information of the uterine cornua [15]. Laparoscopy and direct visualization of the uterus and pathology is helpful when transvaginal sonography fails to differentiate between the above pregnancies.

The estimated recurrence risk of EP ranges from 10\%-27\%, a 5 to 10 fold increase over the risk in general population [16]. The risk factors for various ectopic pregnancies and their recurrence remain the same as previous pelvic/tubal surgery, pelvic inflammatory disease,smoking or past history of IUCD. No separate study has been done to evaluate the factors predisposing to the risk for stump ectopic but it has been found that ipsilateral salpingectomy is a risk factor unique to interstitial pregnancy [13]. Simpson et al. found 46 cases of interstitial pregnancy after ipsilateral salpingectomy with or without cornual resection [17]. The interval between the salpingectomy and the subsequent interstitial pregnancy ranged from 6 months to 13 years.

Various theories have been postulated for the mechanism of recurrent ipsilateral ectopic pregnancy [7,10,14,18]. Reported hypotheses include transperitoneal migration of spermatozoa or embryo through the patent tube to the side of the damaged tube. Another hypothesis is that the oocyte from the normal ovary may be fertilized normally in the patent tube and then later implant in the stump via intrauterine migration. It is also possible that despite the surgical excision of the tube following salpingectomy there is some degree of patency in the remaining interstitial part. Recanalization of isthmic stump allows for fertilization and implantation within this portion of the remnant tube. In our case it is however difficult to prove which mechanism was responsible as there was no corpus luteum evident on ultrasound or laparoscopy.

Management of Ectopic pregnancy can be either expectant or medical management with methotrexate in selected group of patients who fulfil the criteria (RCOG Greentop Guideline). However, laparoscopic salpingectomy or salpingostomy is the preferred approach today (Grade B recommendation RCOG) [19]. Salpingectomy is preferable to salpingostomy if contralateral tube is healthy, as it is associated with lower rates of persistent trophoblastic tissue and subsequent recurrence while having the same intrauterine pregnancy rates [19].

In our case as our patient had hemoperitoneum, she was not a suitable candidate for expectant or medical management. Hence a decision for surgical management was taken. Laparoscopic salpingectomy can be done by various ways using diathermy, newer advanced electrosurgical devices or by using endo-loops or clips. Electrocauterization of the affected fallopian tube and mesosalpinx for hemostasis and excision of the tube is frequently performed. There are studies which show that endo-loop ligation is as good as electrosurgery for hemostasis and it prevents visceral injury related to use of diathermy [20]. The author also claimed that endo-loop ligation is associated with shorter operating time, lower postoperative pain scores and total analgesia. On the other hand, there are case reports demonstrating increased recurrence of ectopic in proximal tubal stump owing to incomplete removal of the tube with endo-loop due to technical difficulty $[6,21,22]$.

Some authors have suggested the use of electrosurgery to excise the proximal tube followed by endo-loop for total salpingectomy to reduce the risk of recurrence of ectopic in tubal stump [6]. Fisher et al suggest that it is fallacious to assume that total salpingectomy is as total as the name implies and there are inherent difficulties to achieve total salpingectomy by using only endoloops.

Although total salpingectomy does not necessarily eradicate all ipsilateral stump ectopics or interstitial ectopics, it certainly decreases an ipsilateral tubal recurrence in proximal or distal stump.Given the rarity of occurrence and uncertain nature of the mechanism, selecting a method for prevention of stump ectopic is difficult. However, a few options may be suggested to decrease the probability of recurrence. When performing the salpingectomy, care should be taken not to leave a long stump. Generally, it is common practice to leave a small tubal stump to minimise the risk of bleeding associated with the isthmic portion of the fallopian tube. However, given the risk of recurrence in those with a history of ectopic pregnancy, most of the authors suggested that this remnant portion should be minimised. Adequate fulguration of the residual stump is advocated to prevent endosalpingoblastosis and potential fistula formation in the stump through which sperm can reach the ovum [23].

\section{Conclusion}

Recurrence of ectopic pregnancy in the remnant tubal stump can have significant clinical consequences. Diagnosis of tubal stump ectopic is difficult and hence there should be high index of suspicion. Even in the near complete resection of the tube in total salpingectomy/ adnexectomy recurrence has been noted. Hence, partial salpingectomy is not recommended. It is not known if the recurrence of ectopic pregnancy in the remnant tubal stump can be prevented. However, all possible measure should be taken to avoid this situation.

\section{References}

1. Boufous S, Quartararo M, Mohsin M, Parker J (2001) Trends in the incidence of ectopic pregnancy in New South Wales between 1990-1998. Australian and New Zealand Journal of Obstetrics and Gynaecology 41: 436-438.

2. Tulandi T (1988) Reproductive performance of women after two tubal ectopic pregnancies. Fertil Steril 50: 164-166. [Crossref]

3. Khan KS, Wojdyla D, Say L, Gülmezoglu AM, Van Look PF (2006) WHO analysis 
of causes of maternal death: a systematic review. Lancet 367: 1066-1074. [Crossref]

4. Adebamowo CA, Fakolujo OA (2000) Second ipsilateral ectopic gestation after total salpingectomy: a case report. Afr J Med Med Sci 29: 63-64. [Crossref]

5. Mathew M, Kumari R, Gowri V (2002) Three consecutive ipsilateral tubal pregnancies. International Journal of Gynaecology and Obstetrics 78: 163-164.

6. Rizos A, Eyong E, Yassin A (2003) Recurrent ectopic pregnancy at the ipsilateral fallopian tube following laparoscopic partial salpingectomy with endo-loop ligation. Journal of Obstetrics and Gynaecology 23: 678-679.

7. Zuzarte R, Khong CC (2005) Recurrent ectopic pregnancy following ipsilateral partial salpingectomy. Singapore Medical Journal 46: 476-478.

8. Okunlola MA, Adesina OA, Adekunle AO (2006) Repeat ipsilateral ectopic gestation: a series of 3 cases, African Journal of Medicine and Medical Sciences 35: 173-175.

9. Tan TL, Elashry A, Tischner I, Jolaoso A (2007) Lightning does strike twice: recurrent ipsilateral tubal pregnancy following partial salpingectomy for ectopic pregnancy. Journal of Obstetrics and Gynaecology 27: 534-535.

10. Milingos DS, Black M, Bain C (2008) Three surgically managed ipsilateral spontaneous ectopic pregnancies. Obstetrics \& Gynecology 112: 458-459.

11. Faleyimu BL, Igberase GO, Momoh MO (2008) Ipsilateral ectopic pregnancy occurring in the stump of a previous ectopic site: a case report. Cases Journal 21: 343.

12. Chou LL, Huang MC (2008) Recurrent ectopic pregnancy after ipsilateral segmental salpingectomy. Taiwanese Journal of Obstetrics and Gynecology 47: 203-205.

13. Lau S, Tulandi T (1999) Conservative medical and surgical management of interstitial ectopic pregnancy. Fertil Steril 72: 207-215. [Crossref]

14. Takeda A, Manabe S, Mitsui T, Nakamura H (2006) Spontaneous ectopic pregnancy occurring in the isthmic portion of the remnant tube after ipsilateral adnexectomy: report of two cases. The Journal of Obstetrics and Gynaecology Research 32: 190-194.

15. Rastogi R, Meena GL, Rastogi N, Rastogi V (2008) Interstitial ectopic pregnancy: A rare and difficult clinicosonographic diagnosis. Journal of Human Reproductive Sciences 1: 80-81.

16. Butts S, Sammel M, Hummel A, Chittams J, Barnhart K (2003) Risk factors and clinical features of recurrent ectopic pregnancy: a case control study. Fertil Steril 80: 1340-1344. [Crossref]

17. Simpson JW, Alford CD, Miller AC (1961) Interstitial pregnancy following homolateral salpingectomy. A report of 6 new cases and review of the literature. Am J Obstet Gynecol 82: 1173-1179. [Crossref]

18. Yano T, Ishida H, Kinoshita T (2009) Spontaneous ectopic pregnancy occurring in the remnant tube after ipsilateral salpingectomy: a report of 2 cases, Reproductive Medicine and Biology 8: 177-179.

19. MRCOG greentop guideline no 21. London: Royal College of Obstetricians and Gynaecologists (RCOG)

20. Lim, Yun-Hsuen, Soon PN, Paul HO Ng, Ay E T, et al. (2007) Laparoscopic salpingectomy in tubal pregnancy: prospective randomized trial using endoloop versus electrocautery. Journal of Obstetrics and Gynaecology Research 33: 855-862.

21. Anwar, Sonia, Talat Uppal (2010) Recurrent viable ectopic pregnancy in the salpingectomy stump. Australasian J Ultrasound Med 13: 37.

22. Fischer S, Keirse M (2009) When salpingectomy is not salpingectomy-ipsilatera recurrence of tubal pregnancy. Obstetrics and Gynaecology International 11:1-4.

23. McCausland A (1982) Endosalpingosis following laparoscopic tubal coagulation as an etiologic factor of ectopic pregnancy. Am J Obstet Gynecol 143:12-24.

Copyright: $\mathbb{C} 2016$ Lakhotia S. This is an open-access article distributed under the terms of the Creative Commons Attribution License, which permits unrestricted use, distribution, and reproduction in any medium, provided the original author and source are credited. 